\title{
A Functional Perspective on the Embryology and Anatomy of the Cerebral Blood Supply
}

\author{
Khaled Menshawi, * Jay P Mohr, Jose Gutierrez \\ Department of Neurology, Columbia University Medical Center, New York, NY, USA
}

The anatomy of the arterial system supplying blood to the brain can influence the development of arterial disease such as aneurysms, dolichoectasia and atherosclerosis. As the arteries supplying blood to the brain develop during embryogenesis, variation in their anatomy may occur and this variation may influence the development of arterial disease. Angiogenesis, which occurs mainly by sprouting of parent arteries, is the first stage at which variations can occur. At day 24 of embryological life, the internal carotid artery is the first artery to form and it provides all the blood required by the primitive brain. As the occipital region, brain stem and cerebellum enlarge; the internal carotid supply becomes insufficient, triggering the development of the posterior circulation. At this stage, the posterior circulation consists of a primitive mesh of arterial networks that originate from projection of penetrators from the distal carotid artery and more proximally from carotid-vertebrobasilar anastomoses. These anastomoses regress when the basilar artery and the vertebral arteries become independent from the internal carotid artery, but their persistence is not uncommon in adults (e.g., persistent trigeminal artery). Other common remnants of embryological development include fenestration or duplication (most commonly of the basilar artery), hypoplasia (typically of the posterior communicating artery) or agenesis (typically of the anterior communicating artery). Learning more about the hemodynamic consequence that these variants may have on the brain territories they supply may help understand better the underlying physiopathology of cerebral arterial remodeling and stroke in patients with these variants.

Keywords Circle of willis; Embryology; Cerebral arteries; Arterial variants; Stroke; Remodeling

\author{
Correspondence: Jose Gutierrez \\ Department of Neurology, Columbia \\ University Medical Center, 710 W 168th \\ Street, New York, NY, 10032, USA \\ Tel: + 1-212-305-1710 \\ Fax: +1-212-305-3741 \\ E-mail: jg3233@cumc.columbia.edu
}

Received: December 18, 2014 Revised: February 26, 2015 Accepted: February 27, 2015

*This work was done while Mr. Menshawi was visiting research fellow at Columbia University Medical Center.

The authors have no financial conflicts of interest.

\section{Introduction}

A large proportion of strokes worldwide are caused by atherosclerosis affecting the arteries that supply blood to the brain. ${ }^{1-4}$ Traditional vascular risk factors such as hypertension, diabetes, hypercholesterolemia and smoking are associated with atherosclerosis. ${ }^{5-8}$ However, geometric patterns of blood flow and the differential shear stress in the arterial wall are important determinants of the localization of atherosclerosis. ${ }^{9-11}$ The arterial geometry is the anatomical correlate of arterial remodeling, it- self a dynamic process driven in part by the needs of the tissue being perfused..$^{12,13}$ This vital link between anatomy and function is evidenced in the embryological development of the circulatory system as well as in the remodeling changes observed in the adult life in response to physiological and pathological stimuli. ${ }^{1417}$

The goal of this review is to describe the embryology of the arteries supplying blood to the brain, their anatomical variants and how these variants might relate to clinical outcomes. 


\section{Embriology of the cerebral circulation}

The development of the vascular system in the embryo happens before the heart starts beating. ${ }^{18}$ It consists of 2 main stages: vasculogenesis and angiogenesis. ${ }^{19}$ Vasculogenesis can be understood as the process by which hemangioblasts are differentiated into angioblasts and angiogenesis refers to the formation of new vessels. ${ }^{18}$ The main mechanism of angiogenesis is sprouting and it is mainly driven by hypoxia/ischemia and related growth factors from the target tissue. ${ }^{18,20,21}$ As more capillaries are formed, the impedance to flow is reduced in larger arteries thus facilitating flow-induced remodeling of the arteries supplying this area. ${ }^{22}$ The development of the circulatory system supplying blood to the brain begins with the formation of the 6 pairs of primitive branchial arch arteries at the $1.3 \mathrm{~mm}$ embryonic stage, subsequently undergoing heavy modifications during development. ${ }^{23,24}$

The internal carotid arteries (ICA) appear during the $3 \mathrm{~mm}$ embryonic stage ( 24 days) from the combination of the $3 \mathrm{rd}$ branchial arch arteries and the distal segments of the paired dorsal aortae (Figure 1). The ventral portion of the 2nd branchial arch disconnects from the dorsal aorta near the origin of the ICA and becomes the ventral pharyngeal artery. Eventually, the ventral pharyngeal artery and the ICA fuse proximally to form the common carotid artery (CCA) and the distal segment of the ventral pharyngeal artery becomes the external carotid artery (ECA) ${ }^{24}$ At $4 \mathrm{~mm}$ stage ( 28 days), the ICA branches off into the anterior and the posterior division. The anterior division initially supplies the optic and olfactory regions through primitive arteries. ${ }^{14,25}$ At a later embryological stage, the anterior division of the ICA will give rise to the anterior cerebral artery (ACA), the middle cerebral artery (MCA), and the anterior choroidal artery (AChA), while the posterior division will produce the (i.e., fetal) posterior cerebral artery (PCA) and the posterior choroidal artery (PChA). ${ }^{14,25}$ Also at this stage the superior cerebellar artery, a branch of the future basilar artery (BA), is the only blood source to the primitive cerebellum. ${ }^{14}$

The occipital lobe and brain stem growth is the initial stimu-

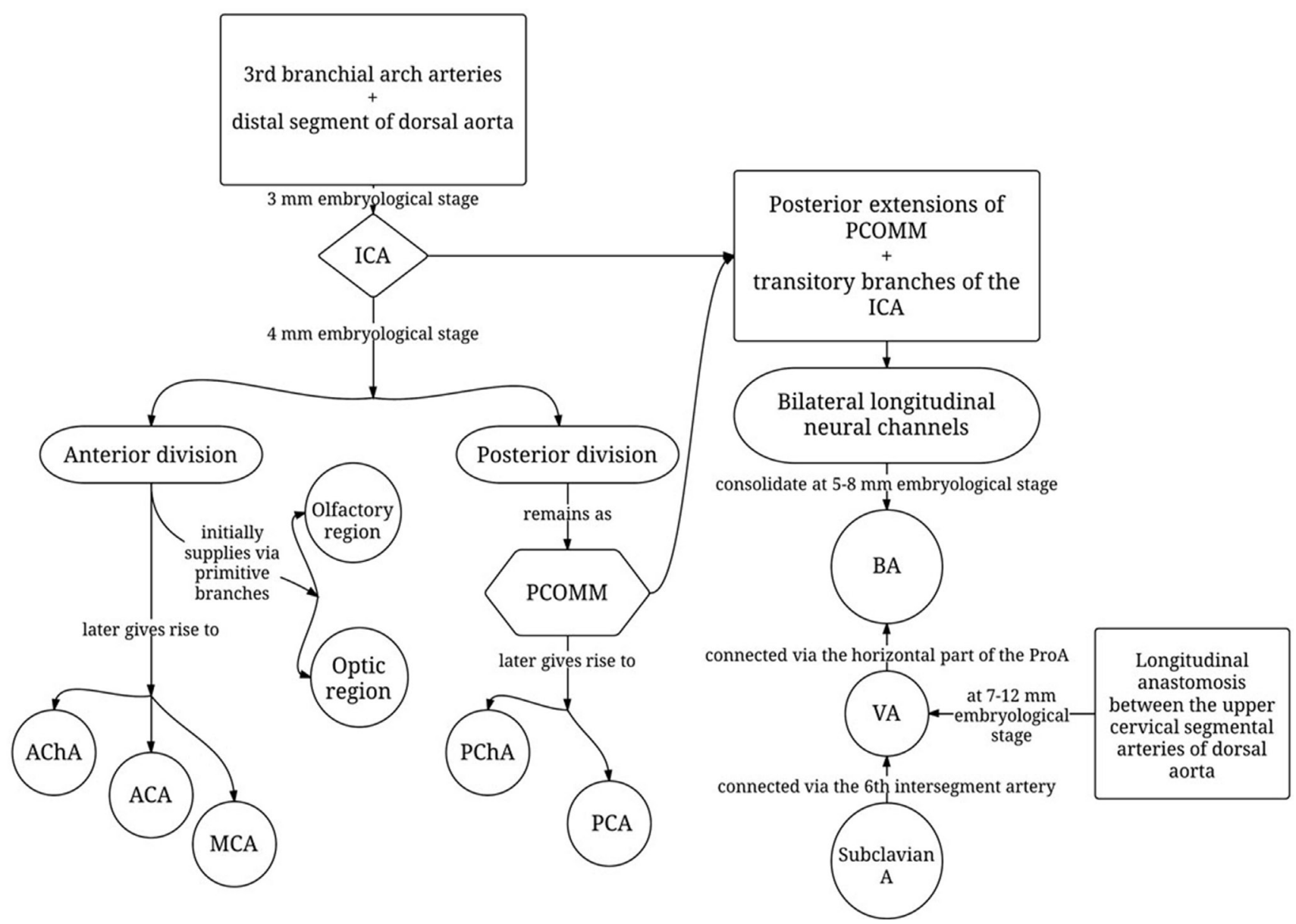

Figure 1. This diagram describes the chronological development of cerebral brain arteries initially with the rise of the internal carotid artery and subsequently with the development of the posterior circulation. The brain differential growth rate is the pacemaker for the development of the cerebral circulatory system with a coupling of the tissue energy demand and the blood supply. 

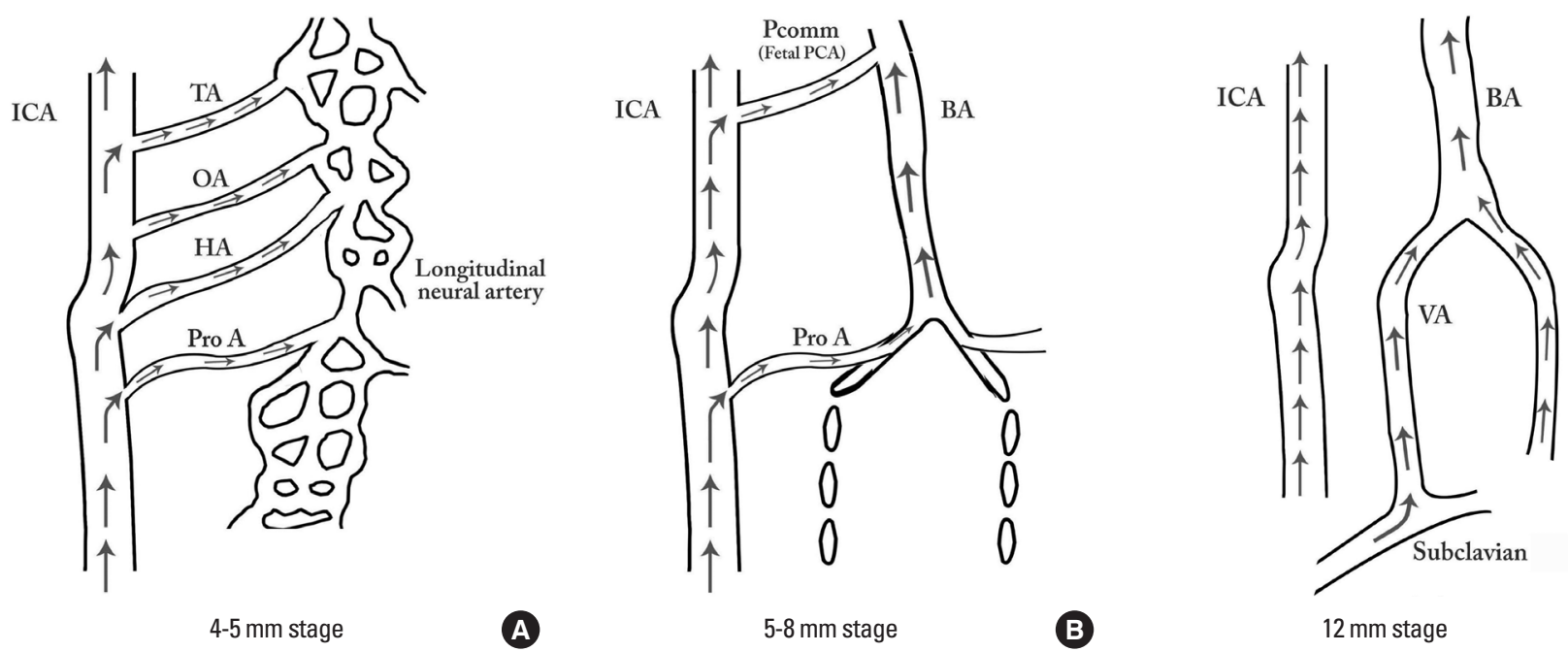

Figure 2. In early phases of development (A), the posterior circulation relies almost entirely from blood supply coming from the anterior circulation through carotidvertebrobasilar anastomoses. Subsequently, as the posterior fossa structures and the occipital lobe growth, the posterior circulation becomes progressively independent from the anterior circulation with obliteration of the anterior-posterior anastomoses from caudal to rostral maintaining in the majority of adult only one connection between the distal basilar arteries with the carotid artery via the posterior communicating artery (B and $\mathrm{C})$.

lus for the formation of the posterior circulation, first with the $\mathrm{BA}$ and later with the vertebral arteries (VA). ${ }^{13}$ At the $4-5-\mathrm{mm}$ embryonic stage, the hindbrain (i.e., future posterior fossa) is supplied by two parallel neural arteries (or channels). These arteries obtain their blood supply from carotid-vertebrobasilar anastomoses given by the trigeminal artery (TA), the otic artery (OA), the hypoglossal artery (HA), and the proatlantal artery (ProA, Figure 2). ${ }^{26,27}$ The BA forms during the $5-8 \mathrm{~mm}$ stage from the consolidation of the neural arteries. The lifespan of the $\mathrm{TA}, \mathrm{OA}$, and the HA is of approximately a week, and when the posterior communicating artery (PCOMM) develops and connects with the distal $\mathrm{BA}$, the three pre-segmental arteries regress. ${ }^{28,29}$ Unlike the TA, OA, and the HA, the ProA persist until the VA are fully developed, and in fact, a segment of the ProA gets incorporated into the V3 segment of the VA and distal portions of occipital artery. ${ }^{26,28}$ At 7 to $12 \mathrm{~mm}$ stage, the VA forms from transverse anastomoses between cervical intersegmental arteries, beginning with the ProA and proceeding downward to the 6th intersegmental artery, which eventually forms the origin of adult VA from the subclavian artery. $23,26,28$

At the 11-12 mm embryological stage (35 days), the development of the MCA is first identified as small buds originating proximal to the ACA on the anterior division of the primitive ICA. ${ }^{23,30}$ Although the MCA is still plexiform and not a true artery, it is the major blood source for the cerebral hemispheres at this stage. ${ }^{15}$ At the $16-18 \mathrm{~mm}$ stage, the MCA becomes more prominent, the plexi fuse into a single artery and further branches pierce the cerebral hemisphere. ${ }^{23}$ At the embryological 18-mm stage, the stem of the ACA gives rise to the olfactory artery. The ACA then continues growing medially towards the contralateral ACA, eventually leading to the formation of the ACOMM at the 21-24 mm embryological stage. ${ }^{14}$ Distally, the small arteries dive into the cerebral cortex in a radially-oriented pattern toward the wall of the developing lateral ventricles forming anastomoses among them. ${ }^{31}$

The posterior aspect of the circle of Willis $(\mathrm{CoW})$ is formed at earlier stages, when the fetal PCA turns into PCOMM, the adult PCA connects with the $\mathrm{BA}$ as branches from the fetal PCA fuse medially to form the distal end of the BA, and the PChA incorporates into the adult PCA. ${ }^{14}$ Consequently, the full development of the ACA and the ACOMM mark the final realization of the adult CoW at the 6-7 weeks embryological stage. ${ }^{14}$

\section{Developmental variants of the cerebral circulation}

As the cerebral vascular tree reaches its typical configuration, the multiple events that occur during the embryological stage might lead to a diverse spectrum of vascular anatomical variants.

\section{Variants affecting all arteries}

\section{Fenestrations and duplications}

Fenestrations of the cerebral arteries are congenital anomalies with a reported prevalence ranging from $0.3 \%$ to $28.0 \%$ depending on the study methods. ${ }^{32-35}$ Fenestration is defined as the splitting of the arterial lumen into two different channels that eventually fuse during their course, restoring the primary vessel ${ }^{36}$ From an anatomic point of view, fenestration should be reserved for variations with a common origin, after which the 

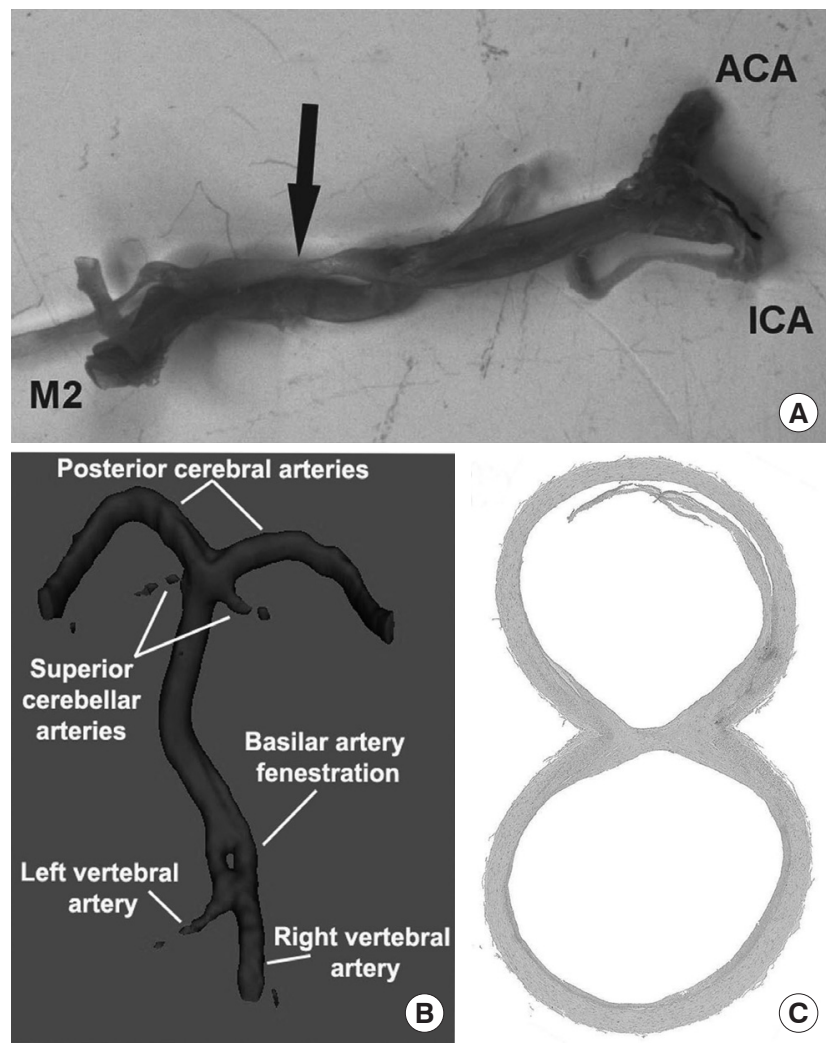

Figure 3. Arterial fenestration can occur in any of the arteries supplying the brain with blood. Fenestrations are defined as splitting of an arterial segment into two separate conducts with a common origin and common convergence prior to a true arterial bifurcation. (A) show an example of a fenestration in the first segment of the middle cerebral artery with convergence prior to the bifurcation into anterior and inferior division. (B) demonstrates the radiological appearance of a basilar artery fenestration, typically located in the most proximal part. (C) shows a cross-sectional view of a basilar artery fenestration (H\&E, $\times 10$ magnification) where it can be observed that the endothelium of both conduits are independent and they are separated by a septum of arterial wall.

artery subsequently splits into two parallel channels that later rejoin and may or not share an adventitial sheath. ${ }^{32,34,37}$ Fenestration should be distinguished from duplication, which is defined as two distinct arteries with separate origins with downstream fusion in a distal arterial segment. ${ }^{36,38}$ Duplicated or fenestrated arteries are the second most common variants (after hypoplastic arteries) and are reportedly more frequent in the anterior circulation. ${ }^{35,39}$ The most commonly duplicated or fenestrated artery is the ACOMM (Figure 3), while fenestrations of the ICA are exceedingly rare. ${ }^{35,40}$ Fenestration or duplications might be due to an incomplete fusion of arteries during development, however some authors believe fenestrations can be also induced by the trans-arterial course of a nerve, a bony structure or enlarged vasa vasorum along the path of the artery. ${ }^{32,34,37,41}$ Except for Digitally Subtracted Angiography (DSA), fenestrations are best visualized with 3D rotational angiography (3DRA). ${ }^{42}$

\section{Hypoplastic arteries}

Cerebral arteries integrating the adult $\mathrm{CoW}$ vary in size. A ratio between certain arteries has been proposed to determine when an artery is smaller than expected. For example, the size of the PCOMM is one half of the size of the PCA, which itself is one half of the BA. ${ }^{14}$ The size of the ACOMM is also one half to two thirds of the ACA size, which itself is one half of the ICA. When the arteries are smaller than usual, they are considered to be hypoplastic or sling-like. ${ }^{43,44}$ In a large autopsy series of normal brains, CoW were classified as normal if they had all the typical arteries integrating in the CoW or deficient if lack of thereof. A "normal" CoW was found in only $52.3 \%$ and the remaining CoW had one or more anomalies. ${ }^{43}$ The most common anomaly was the presence of at least one hypoplastic artery with patent lumina (e.g., $<1 \mathrm{~mm}$. in external diameter, $27.4 \%$ ), which typically was either the PCOMM or the ACOMM. The complete absence of an artery was rarer (up to $2.0 \%$ ) and it typically involved the ACOMM. ${ }^{43}$ In a population-based sample of community-dwellers without stroke, we also reported that a complete CoW was the exception. ${ }^{44}$ The majority of the subjects had at least one hypoplastic artery, which was typically the PCOMM and the posterior circulation was the most likely to have hypoplastic arteries. The difference in the rates noted between direct visualization in autopsy or DSA cases versus brain MRA can be partially attributed to lower accuracy of MRA techniques in distinguishing between a hypoplastic or absent artery. ${ }^{45}$

\section{Specific arterial variants}

After reviewing the literature and synthesizing the data, we proposed a classification of cerebral variants into four groups: a) anomaly of a normally present artery, b) persistence of a normal embryonic artery, and c) visualization of normally hidden anastomoses.

\section{Arteries normally present in the adult CoW Internal carotid artery}

The normal ICA can be divided into multiple segments based on its course. The first segment (i.e., cervical) begins at the CCA bifurcation and ends at entrance into the skull base via the carotid foramen. The second segment (i.e., ascending petrous) extends to the apex of the antero-medial curvature within petrous bone and ends with the origin of the carotico-tympanic artery. The third segment (i.e., horizontal petrous) ends at the skull base in the foramen lacerum. The fourth segment (i.e., ascending cavernous) enters the cavernous sinus and ends with the takeoff of the meningo-hypophyseal trunk where the ICA assumes an anterior-inferior course. The fifth segment (i.e., 
horizontal cavernous) extends from the meningo-hypophyseal trunk to the infero-lateral trunk within the cavernous sinus. The sixth segment (i.e., clinoidal) is bordered proximally by the infero-lateral trunk and distally by the adult OA. The seventh segment lies between the primitive $\mathrm{OA}$ and the $\mathrm{ACA} .{ }^{24,46}$

An aberrant ICA pathway occurs when the ICA deviates from its anatomical landmarks. The most commonly reported aberrant ICA involves the middle ear. ${ }^{47}$ In this variant, the ICA enters the floor of middle ear and then turns forward passing through the middle ear subsequently emerging through the foramen lacerum. Although termed aberrant ICA, the basic pathology in this condition is believed to be hypoplasia or agenesis of the ICA due to persistent collaterals, which substitutes the cervical and vertical petrous portions of the ICA through branches of the ECA. ${ }^{48}$ An aberrant ICA pathway might be accompanied by a persistent stapedial artery. The persistent stapedial artery is a temporary embryonic artery that typically regresses by the third fetal month. The reported prevalence of a persistent stapedial artery is $0.5 \%{ }^{49,50}$ The persistent stapedial artery supplies the middle meningeal artery territory, leading to the absence of the foramen spinosum. Absence of the foramen spinosum is seen in up to $3 \%$ of skull-base CT studies, and it is an indirect sign of a persistent stapedial artery, but it can also be related to an ophthalmic origin of the middle meningeal artery. ${ }^{51,52}$ A true ICA agenesis, aplasia, or hypoplasia is reported in $0.01 \%$ of the population..$^{53,54}$
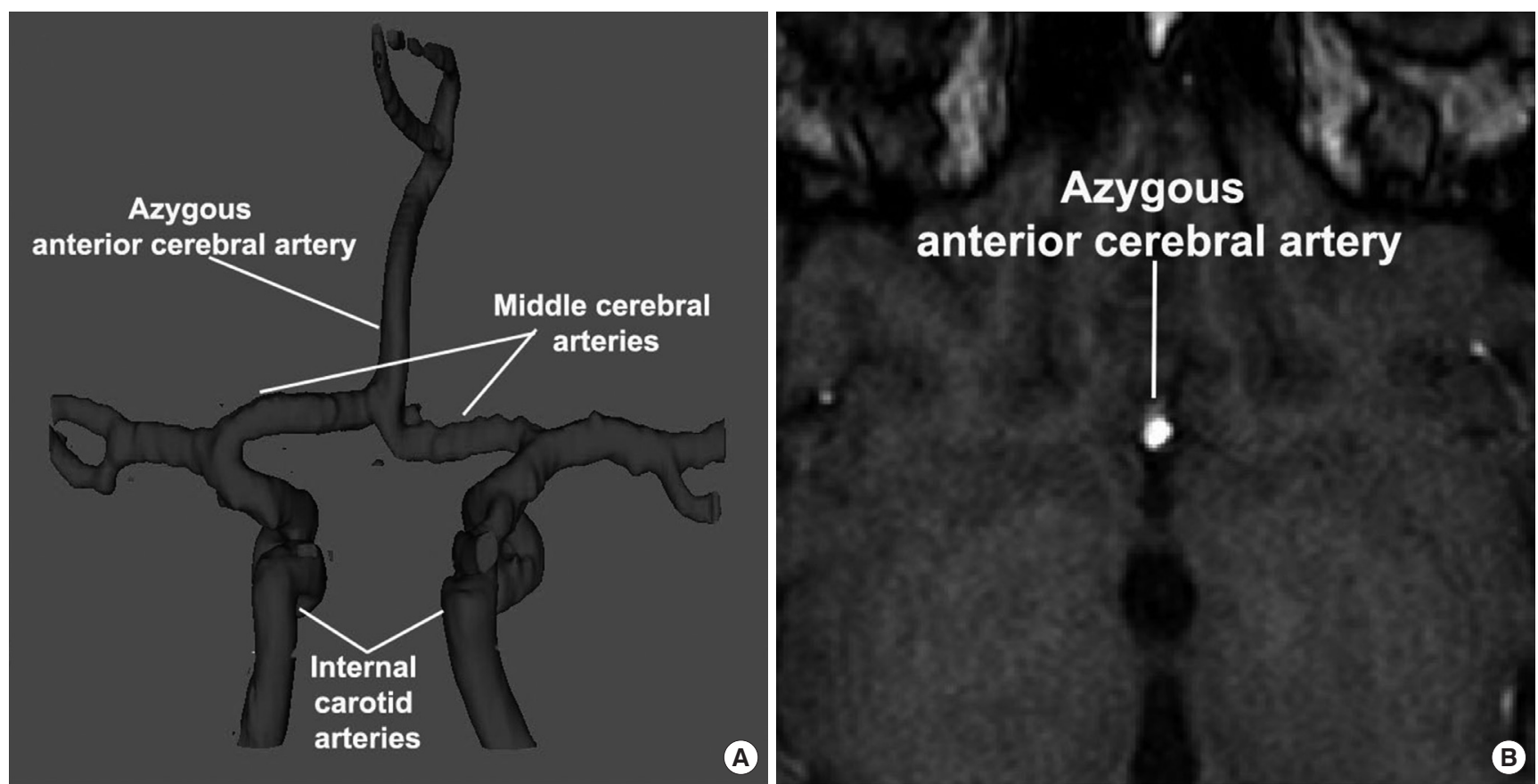

Figure 4. An Azygous anterior cerebral artery is characterized by the fusion of the distal segments of the anterior cerebral artery that later bifurcates to supply the medial aspects of both hemispheres (A). In cross-sectional view of time of flight MRA, a single, typically large anterior cerebral artery can be observed (B). 
persists normally in many vertebrates, but in humans it is considered anomalous and only rarely found in adults $(2.5 \%){ }^{14,60}$

Variants of the ACOMM are common variants in the CoW with a duplication prevalence of $18 \%$ and fenestrations in up to $21 \% .{ }^{60}$ The ACOMM may not appear in angiography, but we cannot be sure about its real absence. Definitive absence of the ACOMM has been found in 5\% of surgical dissections. ${ }^{61,62}$

\section{Basilar artery}

One of the most common embryological variants of the $B A$ is its fenestration, also known as segmental duplication (Figure 3B, C). In the literature, the prevalence of $\mathrm{BA}$ fenestrations ranges from $3.9 \%$ in autopsy reports to $1.1 \%$ in angiographic reports. ${ }^{34,37}$ The fenestration usually occurs more proximal than distal, closer to the vertebrobasilar junction. ${ }^{38}$ The morphological similarities between embryonic and postnatal specimens of BA fenestrations suggest that fenestrations are inborn variants and only rarely undergo further modifications in the adult. ${ }^{63}$

\section{Vertebral artery}

In a large surgical series, variants involving the origin of the VA were not uncommon. ${ }^{64,65}$ The VA normally arises from the upper, posterior surface of subclavian artery 0.5 to $2.0 \mathrm{~cm}$ medial to thyrocervical trunk. Some of the variants of VA origin include a common trunk with the thyrocervical trunk (3.0\%), an aortic origin of the left VA $(2.5 \%$, arising between the left CCA and the left subclavian artery), a right VA arising at the site of bifurcation of the innominate artery into right subclavian and right CCA (1.1\%.), and a right VA as a direct branch of the right CCA $(0.3 \%)$. The segment between the VA origin and the site of entrance to the foramina of the transverse processes is called the V1 segment. In the majority of the cases, the V1 segment ends at the 6 th vertebra (87.5\%), but occasionally it ends at the 5th $(6.6 \%)$, the 7 th $(5.4 \%)$ or less frequently the 4 th vertebra (1.3\%). Other VA variants such as dual (i.e., accessory) VA are rare $(0.7 \%)$.

\section{Posterior cerebral artery}

A fetal origin of the PCA is the most frequent variant involving this artery. It has been reported in between $13 \%-15 \%$ of the population, and it is typically described in only one PCA, but it can also occur in both PCA in up to $0.5 \%$ of the population. ${ }^{39,44}$ More commonly (in up to $10 \%$ of the cases), the P1 segment is hypoplastic, which renders a fetal-like circulation to the occipital lobes, although it is not technically a fetal PCA given the physical link of the PCOMM with the BA..$^{27,66}$ Duplications of the PCA (in either the P1 or P2 segments) are extremely rare (1.0 $\%) .{ }^{61,67}$

\section{Posterior communicating artery}

A hypoplastic PCOMM is overall the most common arterial variant reported in the brain. ${ }^{43,44}$ Duplication of the PCOMM artery is a rare event with a prevalence of $1.8 \%$ in surgical dissection series, and absence of the PCOMM artery is even rarer $(<1.5 \%){ }^{43,68}$ Technically speaking, a fetal PCA by definition is always accompanied by agenesis of the ipsilateral PCOMM, but in fact it is the P1 segment of the PCA that failed to form.

\section{Persistence of normal embryonic arteries: carotid- vertebrobasilar anastomoses}

What causes embryonic arteries to persist in supplying the brain with blood is not entirely clear. In some instances, for example in the case of a persistent TA (see below), it has been noted that there is a delay in the PCOMM development, which would lead to an incomplete support to the distal BA and with it, to a persistent need for these inferior anastomoses to maintain the flow. ${ }^{23}$ Additional arguments in favor of the hypothesis that persistent embryonic arteries exist to make up for the lack appropriate flow from arteries normally present can be found in reports describing the presence of hypoplastic PCOMMs or a hypoplastic ICA with some of these variants. ${ }^{69,70}$

\section{Trigeminal artery}

Persistent TA is the most common of the persistent carotidvertebrobasilar anastomoses, with a reported prevalence of about $0.2 \%(0.1 \%-0.6 \%$, Figure $5 \mathrm{~A}){ }^{26,71,72}$ Instances of persistent TA can be classified in three ways. In type 1 persistent TA, the TA joins the BA between the superior cerebellar artery (SCA) and the anterior inferior cerebellar artery (AICA), the caudal segment of the BA can be hypoplastic and it might show poor filling in angiogram. In type 2 persistent TA, the TA joins the BA above the origin of the SCA and it supplies most of the flow to the SCA. This type is usually accompanied by normal PCOMM supplying most of the PCA flow. The type 3 persistent TA can have an insertion above or below the SCA supplying the SCA and the contralateral PCA while the PCOMM supplies the ipsilateral PCA. In all instances, the VA are usually present and they supply the more inferior aspects of the cerebellum and the lower brain stem. ${ }^{69}$ More rarely, the trigeminal system arising from the ICA supplies the cerebellar arteries directly, bypassing the BA. ${ }^{73}$ This so called "persistent TA variant" is found in up to $0.2 \%$ of angiographic series and it is typically unilateral, although in even rarer instances, it can be found bilaterally $(0.0012 \%){ }^{73,74} \mathrm{~A}$ further subdivision of type 3 persistent TA has been suggested to incorporate the persistent TA variant and name it according to the cerebellar artery arising from the ICA. Persistent TA type 3a, 3b, and $3 c$ are named if the TA sup- 

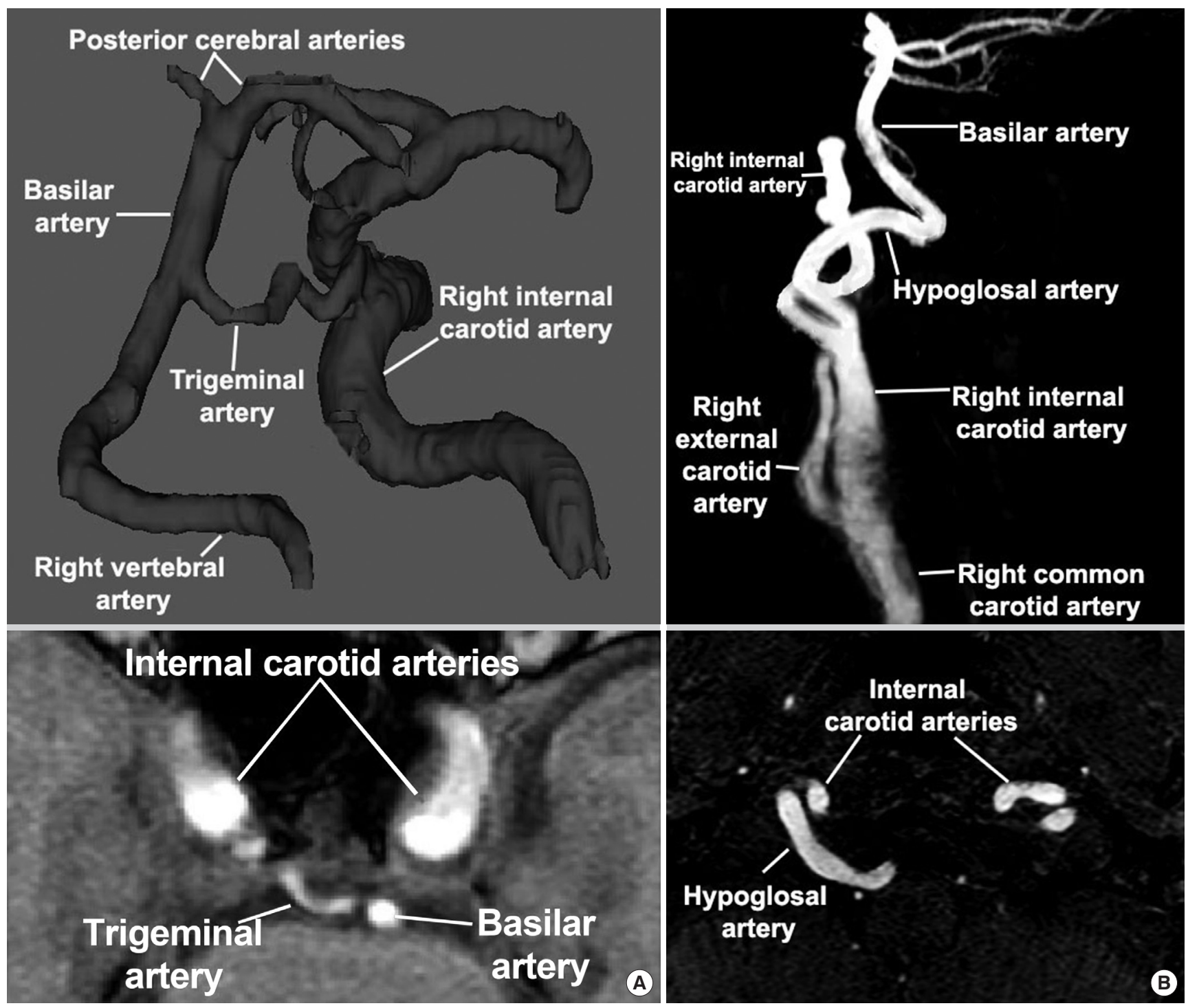

Figure 5. The most common persistent embryonic carotid-vertebrobasilar anastomosis is the trigeminal artery (A). A persistent trigeminal artery consists of a connection between the intracranial internal carotid artery and the basilar artery, usually in the basilar midsegment or distally near the origin of the superior cerebellar arteries. A rarer variant is the persistence of the hypoglossal artery $(B)$, which is distinguished from a persistent proatlantal artery because it enters the skull via the hypoglossal canal rather than the foramen magnum.

plies directly the SCA, AICA, or PICA, respectively, the majority being Type $3 b^{72,75}$

\section{Hypoglossal artery}

The prevalence of a persistent $\mathrm{HA}$ is 8 to 10 times less than the persistent TA with a reported prevalence of $0.02 \%$ to $0.10 \%$ (Figure $5 \mathrm{~A}){ }^{76}$ It arises from the cervical ICA at the $\mathrm{C} 1$ to $\mathrm{C} 3$ level and connects to the BA via the hypoglossal canal (i.e., the anterior condyloid foramen). The HA can be associated with absence of one or both VA, and occasionally of one or two PCOMM. The persistent HA is not identical to its primitive precursor in the embryo. Interestingly, the pathway of the embryonic HA is different from the pathways of the adult persistent HA. The embryonic HA passes medial and anterior to the root of the hypoglossal nerve, while its adult counterpart runs posterior and lateral to it. This suggests that the cranial part of the adult HA was later formed through anastomotic channels with the basal artery. There have been cases where the HA supplies the PICA artery directly, in which case it is called a "persistent $\mathrm{HA}$ variant". ${ }^{28}$

\section{Proatlantal artery}

When the ProA persists, one or both VA are typically hypoplastic. ${ }^{77}$ A persistent ProA can be classified into two types depending on the parent artery. Type 1 arises from the ICA and type 2 arises from the ECA, both entering the skull via the foramen magnum. ${ }^{78}$ The type 1 joint the $\mathrm{V} 4$ segment, while type 2 persistent ProA joins the V3 segment. ${ }^{26,77,79}$ Although persistent 
HAs and persistent ProAs appear similar, there are distinguishing characteristics that can be used to differentiate these two variants. For example, the ProA typically has a suboccipital horizontal pathway that corresponds to the $\mathrm{V} 3$ segment of a normal VA while the HA is more vertically oriented. Furthermore, the ProA enters the skull through the foramen magnum, whereas the $\mathrm{HA}$ enters the skull through the hypoglossal canal. ${ }^{77} \mathrm{Fi}-$ nally, a higher origin is expected for HA arising from the ICA at the level of the $\mathrm{C} 1$ vertebra or $\mathrm{C} 1-\mathrm{C} 2$ interspace, while the origin of ProA is typically at the $\mathrm{C} 2$ or $\mathrm{C} 3$ vertebra. ${ }^{80}$

\section{Otic artery}

Persistence of the otic artery is one of the rarest variants of the carotid-vertebrobasilar anastomoses, and it is usually described as arising from the petrous ICA, then passing through the internal auditory canal to later join the $\mathrm{BA} .^{26}$ There has been one case of an OA giving rise to the PICA. ${ }^{81}$

\section{Visualization of normally hidden anastomoses}

During embryogenesis, multiple connections occur between the ICA, the ECA and the posterior circulation. These anastomoses are not usually seen in conventional angiographic studies due to hemodynamic balance between the involved systems, but they can become visible in the setting of increased intracranial pressure or hemodynamically significant arterial stenoses. ${ }^{82}$ We present here some of the more common anastomoses:

\section{ECA to ophthalmic artery (OphA)}

The three main collaterals route between the OphA (itself a branch of the ICA) and the ECA system are the dorsal nasal artery (a branch of the facial artery), the supraorbital artery (a branch of the superficial temporal artery) and the internal maxillary artery (through proximal and distal branches). ${ }^{83,84}$ The choroidal blush after an ICA injection can be considered an indication of a dominant ICA supply to the eye. Consequently, the lack of choroidal blush with an ICA injection should prompt the investigation of a choroidal blush after an ECA injection, thus supporting an atypical collateral supply to the eye. Occasionally, agenesis of the ICA may be accompanied by the persistence of primitive arteries supplying the eye (i.e., persistence of the stapedial artery). ${ }^{52}$

\section{ECA to cavernous-petrous region of the ICA}

The two main arteries involved in this anastomotic connections include the ascending pharyngeal artery and the internal maxillary artery (through its proximal and distal portions). ${ }^{82}$ The ascending pharyngeal artery arises from the proximal ECA or occasionally, from the proximal segment of the occipital ar- tery. The ascending pharyngeal artery gives off the pharyngeal and the neuromeningeal trunks, which have anastomoses with the ICA through the infero-lateral trunk (via the superior pharyngeal artery) and with the caroticotympanic artery (via the inferior tympanic artery). ${ }^{85}$ Visualization of any of these collateral channels may imply a functional impairment in the ICA, either due to a decreased diameter or to acquired pathology such as stenosis or dissection.

\section{ECA to the posterior circulation}

The most commonly cited anastomosis between the ECA and posterior circulation is the occipital artery, itself a remnant of the ProA. ${ }^{26,27}$ Persistence of the ProA is rare, but the occipital artery retains its anastomotic connection between the ECA and the vertebrobasilar system via the posterior anastomotic radicular branches of the horizontal segment of the occipital artery. ${ }^{82}$ Other anastomotic routes include the posterior descending branch of the HA (a branch of the neuromeningeal trunk of the ascending pharyngeal artery), which supplies the upper cervical vertebral segments and anastomoses with the V3 segment of the VA at the C2-C3 segment and the musculospinal artery, which anastomoses with the VA at the C2-C3 segment and with the ascending and deep cervical arteries. ${ }^{85}$ The ascending cervical artery typically arises from the thyrocervical trunk (a branch of the subclavian artery) or directly from the subclavian artery while the deep cervical artery arises from costocervical trunk. ${ }^{86,87}$ In this context, the musculospinal artery may offer an anastomotic route between extracervical arteries and the posterior circulation in case of disease of the extracranial VA such as atherosclerosis or dissection.

\section{Risk associated with variants in the cerebral circulation}

Although many cerebral arterial variants may be asymptomatic, their recognition is important in patients with cerebrovascular disease and in those who undergo brain imaging for other purposes, as some of these variants might be pathological or turn pathological in a surgical setting.

\section{Arterial geometry and atherosclerosis}

There is a substantial interest in the "geometric risk" for atherosclerosis. ${ }^{9,88-90}$ It is well-accepted that atherosclerotic plaques occur in areas of low or uneven (i.e., oscillatory) wall shear stress. The outer wall of arterial bifurcations (opposite to the flow divider), especially in the presence of obtuse bifurcating angles, is an example of an arterial region consider favorable to the development of atherosclerosis. ${ }^{11,90}$ The blood flow be- 
comes stagnant in some areas of the arterial bifurcation and stagnation makes this region a preferred site for atheroma formation and may predispose to platelet aggregation and thrombus formation. The degree of blood flow stagnation of flow is influenced more by the ratio between the flows of the parentto-daughter arteries than by the diameter ratio between the two. ${ }^{91} \mathrm{~A}$ reduction in parent-to-daughter arterial diameter ratio within a fixed parent-to-daughter flow ratio of 0.50 to 0.69 , however, may lead to a dramatic increment in turbulence, as evidenced by a three-fold plus increment in the Reynold numbers, and thus increasing the oscillatory wall shear stress. ${ }^{90}$ Based on this, dramatic differences in the caliber of branching arteries, presumably acquired during embryogenesis of the brain, could be considered a predisposing anatomy for the development of atherosclerosis.

As discussed above, the primary goal of the developing cerebral arterial system in the embryo is to supply blood to support the rapid and asynchronic brain growth. As long as the flow is delivered, there should not be a deleterious immediate consequence to the brain or the vessel. This statement is supported by the fact that adult individuals who have persistence of fetal arterial configurations in the brains are typically asymptomatic. In the long term, however, individuals with arterial configurations consisting of decreased collaterals routes (e.g., lack of PCOMM or ACOMM) or coexistence of branching arteries with discrepant diameters (i.e., wide diameter ratios), may be at a higher risk of developing atherosclerosis in areas with chronic low or oscillating blood flow.

\section{Carotid bulb bifurcation and atherosclerosis}

In the carotid bifurcation, intima thickening and atheromas rarely occur in the flow divider but are more common in the outer wall of the bifurcation, where low or oscillating wall shear stress is found..$^{2}$ It is less well-established whether the geometry of the bifurcation per se is enough to lead to atherosclerosis. For example, among young individuals ( $24 \pm 4$ years), the inter-individual variability in the CCA/ICA ratio is lower than among older individuals ( $63 \pm 10$ years), suggesting that with aging, the geometry of the carotid bifurcation remodels into more heterogeneous configurations. ${ }^{88}$ This given geometry is influenced by demographic variables such as sex and ethnicity, but also by traditional vascular risk factors. ${ }^{93,94}$ The interplay between a given geometry and vascular risk factors may explain the broader spectrum of disease among older individuals. Nonetheless, considering upfront the ongoing carotid bifurcation remodeling in the in the setting of early atherosclerotic disease, the role that the arterial geometry appears to play a lesser role in the development of carotid atherosclerosis compared with traditional vascular risk factors or aging. ${ }^{9}$

\section{Intracranial arterial geometry and atherosclerosis}

In intracerebral arteries, the larger the interadventitial arterial diameter is, the greater the degree of stenosis noted. After adjustment for artery type (e.g., ICA vs. MCA vs. BA) and artery location (proximal vs. distal segments of the same artery), however, the strength of association between arterial size and stenosis decreases. ${ }^{95}$ This finding suggests that the individual intracranial arterial anatomy may play a role in the development of atherosclerosis independent of arterial size. For example, the degree of intima thickening between the more proximal and distal segment of an artery are relatively similar in the early phases of atherosclerosis, but as the stenosis progresses, the proximal segment of the artery (i.e., immediately preceded by a bifurcation) exhibits greater degrees of stenosis. ${ }^{96}$ In the vertebro-basilar junction, an obtuse confluence angle may predispose to the development of plaques in the apex of the junction (i.e., the inverse flow divider).$^{89}$ It could be argued that the actual geometry of the cerebral arteries changes with advancing atherosclerosis as it is reported in the carotid or coronary arteries, a fact that would make more difficult to disentangle the effects of arterial remodeling in explaining the observed association between geometry and intracranial atherosclerosis. Nonetheless, recent evidence suggests that cerebral arteries may not undergo significant outward remodeling in the setting of arterial, thus making more plausible a geometric risk for atherosclerosis in the cerebral arteries. ${ }^{97}$

Whether the posterior circulation has a higher risk of developing atherosclerosis is unknown. The MCA is typically overrepresented in samples studying symptomatic intracranial large artery atherosclerosis, perhaps due to the likelihood of symptoms upon plaque rupture or erosion in this location. ${ }^{98-100}$ In a large sample of cerebral arteries with predominance of asymptomatic patients, there is less evidence that the MCA is preferentially involved. In the Brain Arterial Remodeling Study (BARS) series, the proximal segment of the supraclinoid ICA and the proximal segments of the VA-V4 segment showed the greatest degree of stenosis. ${ }^{95}$ Determining whether plaque susceptibility and vulnerability for rupture varies by anatomical region may lead to a better understanding of the risk for vascular events based on an individual's arterial anatomy. Achieving this degree of knowledge may advance the effort to develop a personalized approach to prevent the development of intracranial atherosclerosis.

The prospective study of arterial remodeling is challenging. We are not aware of any longitudinal study primary focused on brain arterial anatomy that has included newborns or children 
and follow them into adulthood or late aging. Despite the absence of such a study, it can postulated that individuals born with an arterial geometry favorable for the development of atherosclerosis can more easily, upon the exposure to endothelial injury induced by traditional vascular risk factors, develop atherosclerosis. It should be noted, however, that the "geometric risk for atherosclerosis" is not sine qua non. Consequently, atherosclerosis should not be seen as inevitable in individuals who exhibit these variants. A more aggressive screening and treatment of traditional vascular risk factors may blunt some of the predisposing effects of an "atherosclerosis-enabling" anatomy.

\section{Arterials variants and stroke}

Hypoplastic arteries are probably the most common variants seen in adults. The significance of these variants and has not yet fully validated in population-based studies, but autopsy series have shown that hypoplastic arteries are more commonly found in patients who have had brain infarcts. ${ }^{39}$ As described in direct observation in a large autopsy series, the lumen of hypoplastic arteries is typically patent. ${ }^{14}$ In the absence of proximal large artery stenosis, the communicating arteries seem to affect the final flow volume to the brain only minimally. ${ }^{39,101}$ However, in the setting of large artery occlusion or severe stenosis, the communicating arteries become crucial. It is in this setting that the caliber of the communicating arteries is inversely proportional to risk of stroke as the smaller these communicating arteries are, the lesser the capacity to make up for the reduced or lost flow. Although hypoplastic arteries can be found at birth, there is increasing prevalence of hypoplastic arteries with age and in association with cardiac ischemic disease suggesting that some hypoplastic arteries in adults might undergo hypotrophic, inward remodeling, perhaps in the setting of vascular disease. ${ }^{4,102}$ The persistence of carotid-vertebrobasilar anastomoses occasionally indicates a complete occlusion or agenesis of anterior circulation arteries or the vertebral arteries, which might also compromise the ability of the interconnecting brain arterial anatomy to provide a backup blood supply if needed. ${ }^{34,77}$

It is less well-established whether other variants mentioned here increase the risk of vascular disease. However, it seems important to be aware of the possibility of these variants when assessing an atypical pattern of stroke. For example, a single embolism through an azygous ACA can cause bilateral medial frontal strokes while extracranial carotid stenosis can cause a brain stem stroke through a persistent carotid-vertebrobasilar anastomosis. ${ }^{24,103,104} \mathrm{~A}$ vertebral steal phenomenon may occur in the setting of extracranial carotid artery stenosis because of preferential flow from the vertebrobasilar system to the carotid artery via the ProA. ${ }^{105}$

\section{Arterials variants and aneurysms}

Fenestrations and/or duplication of intracranial arteries have been associated with the development of brain saccular aneurysm. ${ }^{30,106}$ Some plausible explanations for this presumed observation are the fact that smooth muscle cells and collagen are decreased at the proximal and distal end of the duplicated segment and that saccular aneurysm not arising from bifurcations tends to occurs in arterial segments where marked change and obliteration of vessels characterize the course of embryonic development such as the ACA or the VA. ${ }^{14,107}$ Further support for the notion that fenestrations or duplications are associated with arterial wall weakening come from the finding that among patients with non-aneurysmatic, unexplained subarachnoid hemorrhage, up to $95 \%$ of these patients have fenestrations close to the site of hematoma. ${ }^{42}$ However, in a larger series of angiograms the prevalence of fenestrations associated with saccular aneurysms was not higher than the prevalence of saccular aneurysms at the bifurcations of the circle of Willis suggesting that the predilection for aneurysms to form near a fenestration was similar to that of bifurcations. ${ }^{32}$ Occasionally, BA fenestrations might give the false impression of a flow gap in brain MRA and physicians need to be able to interpret this finding in the proper clinical context and consider more accurate diagnostic methods to rule it out. ${ }^{108}$

Azygous ACA has been associated with saccular aneurysm in the same vessel. The hypothesis behind this association is that the increased flow resulting from the fuse $\mathrm{A} 2$ leads to progressive dilatation of the wall. ${ }^{109}$ Additionally, as the azygous ACA can also be found in association with agenesis of corpus callosum, holoprosencephaly, and arteriovenous malformation, a more generalized abnormality of the tissues including the brain and the arteries supplying it cannot be completely ruled out.

\section{Arterial variants and medico-surgical considerations}

Congenital absence of the ICA and hypoplasia of the ICA needs to be distinguished from acquired stenosis. ${ }^{24}$ While an acquired stenosis is found in the setting of vascular disease in older adults or a dissection, hypoplasia of the ICA can be found in all ages. A hypoplastic ICA in all its trajectory, from the CCA to the most distal portion of the intracranial ICA, is usually accompanied by a larger contralateral ICA. In the setting of ear surgery, an aberrant ICA can pose a tremendous risk of perforation or injury if the surgeon is unaware of its existence. ${ }^{110} \mathrm{An}$ aberrant ICA can also mimic glomus tumor, dehiscent jugular bulb, cholesterol granuloma and petrous carotid aneurysm..$^{52,110-112} \mathrm{An}$ incomplete CoW predisposes to cerebral ischemia during transient closure of the ICA in the setting of carotid manipulations and the risk increases more than threefold in the case of contra- 
lateral ICA occlusion. ${ }^{113}$ Extracranial carotid surgery in the presence of any carotid-vertebrobasilar anastomosis might lead to brain stem embolization or ischemia. ${ }^{14}$

A persistent TA has been associated with aneurysms of the CoW, carotid and VA agenesis, facial hemangiomas, PCOMM agenesis, moyamoya disease, and aortic arch vessel anomalies in up to $25 \%$ of the cases. ${ }^{72,115}$ Recognizing the persistent TA is critical during the now-infrequently performed Wada test to avoid embolization or infusion of barbiturates into the posterior fossa. Similarly precautions are advised safely to perform endovascular balloon occlusion tests and open surgical procedures, such as carotid endarterectomy or ECA ligation. ${ }^{24}$ Occasionally, the rupture of a persistent TA-associated aneurysm can lead to the development of carotid-cavernous fistulas. ${ }^{75}$ Glossopharyngeal neuralgia and hypoglossal nerve paralysis may be linked to a persistent $\mathrm{HA}^{29}$

Surgeons unaware of VA variations can encounter fatal hemorrhage during operations such as thyroidectomy or excision of a pharyngeal diverticulum where he can accidentally tear the VA while attempting ligation of the inferior thyroid artery or may cause destruction of any adjacent important structures such as the brachial plexus, thoracic duct, CCA, jugular vein. ${ }^{64}$

\section{Acknowledgments}

To Alperin noninvasive Diagnostics, Inc (Miami, FL) for the use of the 3D MRA volumetric analyses tool to obtain Figures $3 \mathrm{~B}, 4 \mathrm{~A}$, and $5 \mathrm{~A}$.

To the Northern Manhattan Study (NOMAS) funded by NINDS R37 NS029993 (Sacco/Elkind) for allowing the use of MRI images reported in Figures 3B, 4A, and 5A.

To Brianna Avenia-Tapper for her editorial assistance.

\section{References}

1. Ohira T, Shahar E, Chambless LE, Rosamond WD, Mosley TH Jr, Folsom AR. Risk factors for ischemic stroke subtypes: the Atherosclerosis Risk in Communities study. Stroke 2006; 37:2493-2498.

2. Bejot Y, Caillier M, Ben Salem D, Couvreur G, Rouaud O, Osseby GV, et al. Ischaemic stroke subtypes and associated risk factors: a French population based study. J Neurol Neurosurg Psychiatry 2008;79:1344-1348.

3. Gutierrez J, Koch S, Dong C, Casanova T, Modir R, Katsnelson $\mathrm{M}$, et al. Racial and ethnic disparities in stroke subtypes: a multiethnic sample of patients with stroke. Neurol Sci 2014; 35:577-582.

4. Di Tullio MR, Russo C, Jin Z, Sacco RL, Mohr JP, Homma S.
Aortic arch plaques and risk of recurrent stroke and death. Circulation 2009; 119:2376-2382.

5. Feigin V, Carter K, Hackett M, Barber PA, McNaughton H, Dyall L, et al. Ethnic disparities in incidence of stroke subtypes: Auckland Regional Community Stroke Study, 20022003. Lancet Neurol 2006;5:130-139.

6. De Silva DA, Woon FP, Lee MP, Chen CL, Chang HM, Wong MC. Metabolic syndrome is associated with intracranial large artery disease among ethnic Chinese patients with stroke. J Stroke Cerebrovasc Dis 2009;18:424-427.

7. Casella IB, Sotelo FJ, Yamazaki Y, Presti C, Vassoler A, Melo HA. Comparison of common carotid artery intima-media thickness between Brazilian Euro-descendants and Afro-descendants with atherosclerosis risk factors. Clinics (Sao Paulo) 2009;64:657-664.

8. Schneider AT, Kissela B, Woo D, Kleindorfer D, Alwell K, Miller R, et al. Ischemic stroke subtypes: a population-based study of incidence rates among blacks and whites. Stroke 2004; 35:1552-1556.

9. Bijari PB, Wasserman BA, Steinman DA. Carotid bifurcation geometry is an independent predictor of early wall thickening at the carotid bulb. Stroke 2014;45:473-478.

10. Nguyen KT, Clark CD, Chancellor TJ, Papavassiliou DV. Carotid geometry effects on blood flow and on risk for vascular disease. J Biomech 2008;41:11-19.

11. Noren D, Palmer HJ, Frame MD. Predicted wall shear rate gradients in T-type arteriolar bifurcations. Biorheology 2000; 37:325-340.

12. Lazorthes G, Gouazé A, Santini JJ, Lazorthes Y, Laffont J. The modelling of the circle of Willis. Role of compressions of the afferent arterial tracts in the movements of the cervical spine and the cephalic extremity. Neurochirurgie 1971;17:361-378.

13. Burger IM, Siclari F, Gregg L, Gailloud P. Bilateral segmental agenesis of the vertebrobasilar junction: developmental and angiographic anatomy. AJNR Am J Neuroradiol 2007;28: 2017-2022.

14. Padget DH. The circle of Willis: its embryology and anatomy. In: Dandy WE. Intracranial Arterial Aneurysm. New York: Comstock, 1945;74-85.

15. Padget D. The circle of Willis: its embryology and anatomy. In: Dandy WE. Intracranial Arterial Aneurysms. Ithaca, N. Y.: Comstock Publishing Company, Inc., Cornell University, 1944;67-90.

16. Gibbons GH, Dzau VJ. The emerging concept of vascular remodeling. N Engl J Med 1994;330:1431-1438.

17. Glagov S, Weisenberg E, Zarins CK, Stankunavicius R, Kolettis GJ. Compensatory enlargement of human atherosclerotic coronary arteries. N Engl J Med 1987;316:1371-1375. 
18. Plate KH. Mechanisms of angiogenesis in the brain. J Neuropathol Exp Neurol 1999;58:313-320.

19. Leblanc GG, Golanov E, Awad IA, Young WL; Biology of Vascular Malformations of the Brain NINDS Workshop Collaborators. Biology of vascular malformations of the brain. Stroke 2009;40:e694-e702.

20. Beck L Jr, D’Amore PA. Vascular development: cellular and molecular regulation. FASEB J 1997;11:365-373.

21. Risau W. Mechanisms of angiogenesis. Nature 1997;386:671674.

22. Faraci FM, Heistad DD. Regulation of large cerebral arteries and cerebral microvascular pressure. Circ Res 1990;66:8-17.

23. Paget DH. The development of the cranial arteries in the human embryo. Contrib Embryol 1948;32:205-262.

24. Kathuria S, Gregg L, Chen J, Gandhi D. Normal cerebral arterial development and variations. Semin Ultrasound CT MR 2011;32:242-251.

25. Kier LE. Section I. Fetal cerebral arteries: a phylogenetic and ontogenic study. In: Newton TH, Potts DG. Radiology of the Skull and Brain. Saint Louis: Mosby, 1971;1089-1130.

26. Luh GY, Dean BL, Tomsick TA, Wallace RC. The persistent fetal carotid-vertebrobasilar anastomoses. AJR Am J Roentgenol 1999; 172:1427-1432.

27. Caldemeyer KS, Carrico JB, Mathews VP. The radiology and embryology of anomalous arteries of the head and neck. AJR Am J Roentgenol 1998;170:197-203.

28. Brismar J. Persistent hypoglossal artery, diagnostic criteria. Report of a case. Acta Radiol Diagn (Stockh) 1976;17:160166.

29. Yilmaz E, Ilgit E, Taner D. Primitive persistent carotid-basilar and carotid-vertebral anastomoses: a report of seven cases and a review of the literature. Clin Anat 1995;8:36-43.

30. LaBorde DV, Mason AM, Riley J, Dion JE, Barrow DL. Aneurysm of a duplicate middle cerebral artery. World Neurosurg 2012;77:201.e1-4.

31. Bär T. The vascular system of the cerebral cortex. Adv Anat Embryol Cell Biol 1980;59:I-VI,1-62.

32. Sanders WP, Sorek PA, Mehta BA. Fenestration of intracrani$\mathrm{al}$ arteries with special attention to associated aneurysms and other anomalies. AJNR Am J Neuroradiol 1993;14:675-680.

33. Teal JS, Rumbaugh CL, Bergeron RT, Segall HD. Angiographic demonstration of fenestrations of the intradural intracranial arteries. Radiology 1973;106:123-126.

34. Vasović L, Trandafilović M, Jovanović I, Ugrenović S, Antović A, Karadžić R, et al. Human basilar artery abnormalities in the prenatal and postnatal period. World Neurosurg 2013;79: 593.e15-23.

35. van Rooij SB, van Rooij WJ, Sluzewski M, Sprengers ME.
Fenestrations of intracranial arteries detected with 3D rotational angiography. AJNR Am J Neuroradiol 2009;30:13471350.

36. Polguj M, Podgórski M, Jędrzejewski K, Topol M, Majos A. Fenestration and duplication of the vertebral artery: the anatomical and clinical points of view. Clin Anat 2013;26:933-943.

37. Parmar H, Sitoh YY, Hui F. Normal variants of the intracranial circulation demonstrated by MR angiography at 3T. Eur J Radiol 2005;56:220-228.

38. Lesley WS, Dalsania HJ. Double origin of the posterior inferior cerebellar artery. AJNR Am J Neuroradiol 2004;25:425-427.

39. Alpers BJ, Berry RG. Circle of Willis in cerebral vascular disorders. The anatomical structure. Arch Neurol 1963;8:398-402.

40. Rennert J, Ullrich WO, Schuierer G. A rare case of supraclinoid internal carotid artery (ICA) fenestration in combination with duplication of the middle cerebral artery (MCA) originating from the ICA fenestration and an associated aneurysm. Clin Neuroradiol 2013;23:133-136.

41. Finlay HM, Canham PB. The layered fabric of cerebral artery fenestrations. Stroke 1994;25:1799-1806.

42. Hudák I, Lenzsér G, Lunenkova V, Dóczi T. Cerebral arterial fenestrations: a common phenomenon in unexplained subarachnoid haemorrhage. Acta Neurochir (Wien) 2013;155: 217-222.

43. Alpers BJ, Berry RG, Paddison RM. Anatomical studies of the circle of Willis in normal brain. AMA Arch Neurol Psychiatry 1959;81:409-418.

44. Gutierrez J, Sultan S, Bagci A, Rundek T, Alperin N, Elkind MS, et al. Circle of Willis configuration as a determinant of intracranial dolichoectasia. Cerebrovasc Dis 2013;36:446-453.

45. Stock KW, Wetzel S, Kirsch E, Bongartz G, Steinbrich W, Radue EW. Anatomic evaluation of the circle of Willis: MR angiography versus intraarterial digital subtraction angiography. AJNR Am J Neuroradiol 1996;17:1495-1499.

46. Lasjaunias P, Santoyo-Vazquez A. Segmental agenesis of the internal carotid artery: angiographic aspects with embryological discussion. Anat Clin 1984;6:133-141.

47. Willinsky R, Lasjaunias P, Berenstein A. Intracavernous branches of the internal carotid artery (ICA). Comprehensive review of their variations. Surg Radiol Anat 1987;9:201-215.

48. Jacobsson M, Davidsson A, Hugosson S, Tjellström A, Svendsen P. Aberrant intratympanic internal carotid artery: a potentially hazardous anomaly. J Laryngol Otol 1989;103: 1202-1205.

49. Moreano EH, Paparella MM, Zelterman D, Goycoolea MV. Prevalence of facial canal dehiscence and of persistent stapedial artery in the human middle ear: a report of 1000 temporal bones. Laryngoscope 1994;104:309-320. 
50. Lasjaunias P, Moret J, Manelfe C, Théron J, Hasso T, Seeger J. Arterial anomalies at the base of the skull. Neuroradiology 1977;13:267-272.

51. Maiuri F, Donzelli R, de Divitiis O, Fusco M, Briganti F. Anomalous meningeal branches of the ophthalmic artery feeding meningiomas of the brain convexity. Surg Radiol Anat 1998;20:279-284.

52. Celebi I, Oz A, Yildirim H, Bankeroglu H, Basak M. A case of an aberrant internal carotid artery with a persistent stapedial artery: association of hypoplasia of the A1 segment of the anterior cerebral artery. Surg Radiol Anat 2012;34:665-670.

53. Given CA 2nd, Huang-Hellinger F, Baker MD, Chepuri NB, Morris PP. Congenital absence of the internal carotid artery: case reports and review of the collateral circulation. AJNR Am J Neuroradiol 2001;22:1953-1959.

54. Chen CJ, Chen ST, Hsieh FY, Wang LJ, Wong YC. Hypoplasia of the internal carotid artery with intercavernous anastomosis. Neuroradiology 1998;40:252-254.

55. Watanabe T, Togo M. Accessory middle cerebral artery. Report of four cases. J Neurosurg 1974;41:248-251.

56. Crompton MR. The pathology of ruptured middle-cerebral aneurysms with special reference to the differences between the sexes. Lancet 1962;2:421-425.

57. Teal JS, Rumbaugh CL, Bergeron RT, Segall HD. Anomalies of the middle cerebral artery: accessory artery, duplication, and early bifurcation. Am J Roentgenol Radium Ther Nucl Med 1973; 118:567-575.

58. Murai Y, Ikeda Y, Sato H, Yamamoto Y, Teramoto A. Anomalous internal carotid anastomosis to contralateral anterior cerebral artery. Can J Neurol Sci 2005;32:359-360.

59. LeMay M, Gooding CA. The clinical significance of the azygos anterior cerebral artery (A.C.A.). Am J Roentgenol Radium Ther Nucl Med 1966;98:602-610.

60. Perlmutter D, Rhoton AL Jr. Microsurgical anatomy of the anterior cerebral-anterior communicating-recurrent artery complex. J Neurosurg 1976;45:259-272.

61. Dimmick SJ, Faulder KC. Normal variants of the cerebral circulation at multidetector CT angiography. Radiographics 2009;29:1027-1043.

62. Rhoton AL Jr, Saeki N, Perlmutter D, Zeal A. Microsurgical anatomy of common aneurysm sites. Clin Neurosurg 1979; 26:248-306.

63. Vasović L, Milenković Z, Pavlović S. Comparative morphological variations and abnormalities of circles of Willis: a minireview including two personal cases. Neurosurg Rev 2002;25: 247-251.

64. Daseler EH, Anson BJ. Surgical anatomy of the subclavian artery and its branches. Surg Gynecol Obstet 1959;108:149-174.
65. Newton TH, Potts DG. Radiology of the Skull and Brain. Saint Louis: Mosby, 1971.

66. Van Overbeeke JJ, Hillen B, Tulleken CA. A comparative study of the circle of Willis in fetal and adult life. The configuration of the posterior bifurcation of the posterior communicating artery. J Anat 1991;176:45-54.

67. Caruso G, Vincentelli F, Rabehanta P, Giudicelli G, Grisoli F. Anomalies of the P1 segment of the posterior cerebral artery: early bifurcation or duplication, fenestration, common trunk with the superior cerebellar artery. Acta Neurochir (Wien) 1991;109:66-71.

68. Sahni D, Jit I, Lal V. Variations and anomalies of the posterior communicating artery in Northwest Indian brains. Surg Neurol 2007; 68:449-453.

69. Saltzman GF. Patent primitive trigeminal artery studied by cerebral angiography. Acta Radiol 1959;51:329-336.

70. Okuno T, Nishiguchi T, Hayashi S, Miyamoto K, Terashita T, Itakura T, et al. A case of carotid superior cerebellar artery anastomosis associated with bilateral hypoplasia of the internal carotid artery represented as the rupture of posterior cerebral artery-posterior communicating artery aneurysm. No Shinkei Geka 1988;16:1211-1217.

71. Raphaeli G, Bandeira A, Mine B, Brisbois D, Lubicz B. A rare variant of persistent trigeminal artery: cavernous carotid-cerebellar artery anastomosis--a case report and a systematic review. Cerebellum 2009;8:445-447.

72. Ali S, Radaideh MM, Shaibani A, Russell EJ, Walker MT. Persistent trigeminal artery terminating in the posterior inferior cerebellar artery: case report. Neurosurgery 2008;62:E746E748.

73. Uchino A. Bilateral persistent trigeminal artery variants diagnosed by MR angiography. Cerebellum 2011;10:745-747.

74. Siqueira M, Piske R, Ono M, Marino Júnior R. Cerebellar arteries originating from the internal carotid artery. AJNR Am J Neuroradiol 1993;14:1229-1235.

75. McKenzie JD, Dean BL, Flom RA. Trigeminal-cavernous fistula: Saltzman anatomy revisited. AJNR Am J Neuroradiol 1996;17:280-282.

76. Oelerich M, Schuierer G. Primitive hypoglossal artery: demonstration with digital subtraction-, MR- and CT angiography. Eur Radiol 1997; 7:1492-1494.

77. Gumus T, Onal B, Ilgit ET. Bilateral persistence of type 1 proatlantal arteries: report of a case and review of the literature. AJNR Am J Neuroradiol 2004;25:1622-1624.

78. Lasjaunias P, Théron J, Moret J. The occipital artery. Anatomy--normal arteriographic aspects--embryological significance. Neuroradiology 1978;15:31-37.

79. Guerri-Guttenberg RA. Fetal carotid-vertebrobasilar anasto- 
moses: persistent hypoglossal artery associated with further variations of the circle of Willis. Surg Radiol Anat 2009;31: 311-315.

80. Anderson RA, Sondheimer FK. Rare carotid-vertebrobasilar anastomoses with notes on the differentiation between proatlantal and hypoglossal arteries. Neuroradiology 1976;11:113118.

81. Tomsick TA, Lukin RR, Chambers AA. Persistent trigeminal artery: unusual associated abnormalities. Neuroradiology 1979; $17: 253-257$.

82. Geibprasert S, Pongpech S, Armstrong D, Krings T. Dangerous extracranial-intracranial anastomoses and supply to the cranial nerves: vessels the neurointerventionalist needs to know. AJNR Am J Neuroradiol 2009;30:1459-1468.

83. Lasjaunias P, ter Brugge KG, Berenstein A. Surgical Neuroangiography: Vol. 3: Clinical and Interventional Aspects in Children. Springer, 2007.

84. Hayreh SS. Orbital vascular anatomy. Eye (Lond) 2006;20: 1130-1144.

85. Hacein-Bey L, Daniels DL, Ulmer JL, Mark LP, Smith MM, Strottmann JM, et al. The ascending pharyngeal artery: branches, anastomoses, and clinical significance. AJNR Am J Neuroradiol 2002;23:1246-1256.

86. Lischka M, Krammer E, Rath T, Riedl M, Ellböck E. The human thyrocervical trunk: configuration and variability reinvestigated. Anat Embryol (Berl) 1982;163:389-401.

87. Huntoon MA. Anatomy of the cervical intervertebral foramina: vulnerable arteries and ischemic neurologic injuries after transforaminal epidural injections. Pain 2005; 117:104-111.

88. Thomas JB, Antiga L, Che SL, Milner JS, Steinman DA, Spence JD, et al. Variation in the carotid bifurcation geometry of young versus older adults: implications for geometric risk of atherosclerosis. Stroke 2005;36:2450-2456.

89. Ravensbergen J, Krijger JK, Hillen B, Hoogstraten HW. The influence of the angle of confluence on the flow in a vertebrobasilar junction model. J Biomech 1996;29:281-299.

90. Karino T, Goldsmith HL. Particle flow behavior in models of branching vessels. II. Effects of branching angle and diameter ratio on flow patterns. Biorheology 1985;22:87-104.

91. Pinchak AC, Ostrach S. Blood flow in branching vessels. J Appl Physiol 1976;41:646-658.

92. Ku DN, Giddens DP, Zarins CK, Glagov S. Pulsatile flow and atherosclerosis in the human carotid bifurcation. Positive correlation between plaque location and low oscillating shear stress. Arteriosclerosis 1985;5:293-302.

93. Schulz UG, Rothwell PM. Sex differences in carotid bifurcation anatomy and the distribution of atherosclerotic plaque. Stroke 2001;32:1525-1531.
94. Ruan L, Chen W, Srinivasan SR, Sun M, Wang H, Toprak A, et al. Correlates of common carotid artery lumen diameter in black and white younger adults: the Bogalusa Heart Study. Stroke 2009;40:702-707.

95. Gutierrez J, Rosoklija G, Murray J, Chon C, Elkind MS, Goldman J, et al. A quantitative perspective to the study of brain arterial remodeling of donors with and without HIV in the Brain Arterial Remodeling Study (BARS). Front Physiol 2014;5:56.

96. Gutierrez J, Elkind M, Virmani R, Goldman J, Lawrence H, Morgello S, et al. A pathological perspective on the natural history of cerebral atherosclerosis. Int J Stroke 2015. doi: 10.1111/ijs.12496.

97. Gutierrez J, Goldman J, Honig LS, Elkind MSV, Morgello S, Marshall RS. Determinants of cerebrovascular remodeling: Do large brain arteries accommodate stenosis? Atherosclerosis 2014;235:371-379.

98. Chimowitz MI, Lynn MJ, Derdeyn CP, Turan TN, Fiorella D, Lane BF, et al. Stenting versus aggressive medical therapy for intracranial arterial stenosis. N Engl J Med 2011;365:9931003.

99. Chimowitz MI, Lynn MJ, Howlett-Smith H, Stern BJ, Hertzberg VS, Frankel MR, et al. Comparison of warfarin and aspirin for symptomatic intracranial arterial stenosis. $N$ Engl J Med 2005;352:1305-1316.

100. Sacco RL, Kargman DE, Gu Q, Zamanillo MC. Race-ethnicity and determinants of intracranial atherosclerotic cerebral infarction. The Northern Manhattan Stroke Study. Stroke 1995; 26:14-20.

101. Alastruey J, Parker KH, Peiró J, B yrd SM, Sherwin SJ. Modelling the circle of Willis to assess the effects of anatomical variations and occlusions on cerebral flows. J Biomech 2007;40: 1794-1805.

102. El-Barhoun EN, Gledhill SR, Pitman AG. Circle of Willis artery diameters on MR angiography: an Australian reference database. J Med Imaging Radiat Oncol 2009;53:248-260.

103. Preul M, Tampieri D, Leblanc R. Giant aneurysm of the distal anterior cerebral artery: associated with an anterior communicating artery aneurysm and a dural arteriovenous fistula. Surg Neurol 1992;38:347-352.

104. Niizuma H, Kwak R, Uchida K, Suzuki J. Aneurysms of the azygos anterior cerebral artery. Surg Neurol 1981;15:225-228.

105. Parkinson D, Reddy V, Ross RT. Congenital anastomosis between the vertebral artery and internal carotid artery in the neck. Case report. J Neurosurg 1979;51:697-699.

106. Campos J, Fox AJ, Viñuela F, Lylyk P, Ferguson GG, Drake CG, et al. Saccular aneurysms in basilar artery fenestration. AJNR Am J Neuroradiol 1987;8:233-236. 
107. Fujimura M, Sugawara T, Higuchi H, Oku T, Seki H. A ruptured aneurysm at the distal end of the basilar artery fenestration associated with multiple fenestrations of the vertebrobasilar system: case report. Surg Neurol 1997;47:469-472.

108. Scherer A, Siebler M, Aulich A. Virtual arterial endoscopy as a diagnostic aid in a patient with basilar artery fenestration and thromboembolic pontine infarct. AJNR Am J Neuroradiol 2002;23:1237-1239.

109. Glasscock ME 3rd, Seshul M, Seshul MB Sr. Bilateral aberrant internal carotid artery case presentation. Arch Otolaryngol Head Neck Surg 1993;119:335-339.

110. Silbergleit R, Quint DJ, Mehta BA, Patel SC, Metes JJ, Noujaim SE. The persistent stapedial artery. AJNR Am J Neuroradiol 2000;21:572-577.

111. Oates JW, McAuliffe W, Coates HL. Management of pseudoaneurysm of a lateral aberrant internal carotid artery. Int $J \mathrm{Pe}$ - diatr Otorhinolaryngol 1997;42:73-79.

112. Moret J, Delvert JC, Bretonneau CH, Lasjaunias P, de Bicĉtre $\mathrm{CH}$. Vascularization of the ear: normal-variations-glomus tumors. J Neuroradiol 1982;9:209-260.

113. Manninen H, Mäkinen K, Vanninen R, Ronkainen A, Tulla $\mathrm{H}$. How often does an incomplete circle of Willis predispose to cerebral ischemia during closure of carotid artery? Postmortem and clinical imaging studies. Acta Neurochir (Wien) 2009; 151:1099-1105.

114. Woodcock RJ, Cloft HJ, Dion JE. Bilateral type 1 proatlantal arteries with absence of vertebral arteries. AJNR Am J Neuroradiol 2001;22:418-420.

115. Okahara M, Kiyosue H, Mori H, Tanoue S, Sainou M, Nagatomi $\mathrm{H}$. Anatomic variations of the cerebral arteries and their embryology: a pictorial review. Eur Radiol 2002;12:2548-2561. 